\title{
Can Children Learn Creativity From A Social Robot?
}

\author{
Safinah Ali \\ MIT Media Lab \\ Cambridge, USA \\ safinah@media.mit.edu
}

\author{
Tyler Moroso \\ MIT \\ Cambridge, USA \\ tlmoroso@mit.edu
}

\author{
Cynthia Breazeal \\ MIT Media Lab \\ Cambridge, USA \\ cynthiab@media.mit.edu
}

\begin{abstract}
Children's creativity contributes to their learning outcomes and personal growth. Standardized measures of creative thinking reveal that as children enter elementary school, their creativity drops. In this work, we evaluated whether a social robotic peer can help 6-10-year-old children think creatively by demonstrating creative behavior. We designed verbal and non-verbal behaviors of the social robot that constitute interaction patterns for artificial creativity. 51 participants played the Droodle Creativity Game with the robot to generate creative titles for ambiguous images. One group of participants interacted with the creative robot, and one group interacted with the non-creative robot. Participants that interacted with the creative robot generated significantly higher number of Droodle titles, expressed greater variety in titles, and scored higher on the Droodles' creativity. We observe that children can model a social robotic peer's creativity, and hence inform robot interaction patterns for artificial creativity that can foster creativity in children.
\end{abstract}

\section{Author Keywords}

Creativity Support Tools; Child-Robot Interaction; Interaction Design

\section{ACM Classification Keywords}

- Applied computing Psychology - Computer systems organization $\sim$ Robotics $\bullet$ Human-centered computing $\sim \mathrm{HCI}$ design and evaluation methods

\section{INTRODUCTION}

Developmental benefits of creativity in children are not unknown. Creativity has shown to facilitate problem solving, adaptability, self-expression and health [25]. Creative thinking has shown to have both social and economic benefits [15]. Research has elaborated the benefits of integrating creative skills into the educational institutions' curricula, and increased learning benefits [37]. As we move from industrialized economies to creative economies,

\footnotetext{
Permission to make digital or hard copies of all or part of this work for personal or classroom use is granted without fee provided that copies are not made or distributed for profit or commercial advantage and that copies bear this notice and the full citation on the first page. Copyrights for components of this work owned by others than ACM must be honored. Abstracting with credit is permitted. To copy otherwise, or republish, to post on servers or to redistribute to lists, requires prior specific permission and/or a fee. Request permissions from Permissions@acm.org.
}

C\&C '19, June 23-26, 2019, San Diego, CA, USA

(C) 2019 Association for Computing Machinery.

ACM ISBN 978-1-4503-5917-7/19/06...\$15.00

https://doi.org/10.1145/3325480.3325499 creativity is a crucial ability for the workplace. Standardized creativity measures have previously demonstrated that as children progress from kindergarten to elementary school, their creativity drops. As observed by the Torrance Test of Creative Thinking (TTCT), there is especially a slump in creativity in grade four (ages 8-10) [43, 45, 40]. It has been suggested that academic expectations from children, and structured curricula that allow little space for play might lead to this fourth-grade slump [2]. These structured school curricula lead to reduced divergent thinking and imagination, and we lose benefits of the kindergarten style play based learning strategies that promote creative thinking. This is especially a concern for a generation of children growing up today in the era of Artificial Intelligence where repetitive, rule driven jobs shift from humans to computers and the technology that children interact with changes rapidly. In this ever-changing world, fostering creativity and imagination can help them succeed.

While there are several frameworks for defining what qualifies as creative, and associated thinking behaviors such as generating novel ideas, divergent thinking, lateral thinking, making unusual connections [4, 16, 25], for the purpose of this work, we limit the definition of creativity to the ability to generate fluent ideas that have novelty and value [4].

Creativity in classrooms is often influenced by extrinsic factors, such as social interactions with peers and teachers [22]. Creativity is influenced by immediate classroom environment, and fostered when activities are presented in permissive and game-like fashion [46]. Teachers also act as potential models for creativity [37]. Artificial embodied agents such as social robots have been used as effective pedagogical tools for young children leading to both cognitive and affective gains. Social robots offer an affordable and scalable way to offer personalized support to learners. Previous work has demonstrated the learning benefits of physically co-present robots versus video displayed agents [49]. As these robots become more accessible, and increasingly enter classroom and domestic environment, there arises a need to be mindful about designing the robots' social behaviors, such that, they not only lead to cognitive gains in children, but also behavioral gains such as curiosity, mindset, persistence, engagement, and creativity. Previous studies have demonstrated how children model a robotic peer's learning behaviors such as curiosity, perseverance and growth mindset [31]. In this work, we explore if interacting with social robots that exhibit 
creative behaviors can help children think more creatively. We designed a Child-Robot Interaction where children play a game that affords creativity, with the social robot Jibo, while the robot exhibits a creative or a non-creative gameplay behavior. The goal of this study was to analyze if children model the robot's creativity.

In order to measure children's creativity, we employed the Droodle Creativity Game, inspired from the Droodle Creativity Task, which is a verbal creativity task that draws upon people's ability to use language in witty and creative ways (Droodle titles) to describe an abstract image or figure (Droodle) [24]. We designed a game-like Child Robot Interaction where the robot and the child take turns to generate Droodle titles. We recruited 51 participants in the 6-10 year old age group that played 5 rounds of the Droodle Creativity Game with Jibo. Participants were divided in two balanced groups based on their pre-test creativity measures (as measured by the standardized Torrance Test of Creative Thinking test), age and gender. One group interacted with the robot that exhibits creative behaviors, that is, chooses more number of ideas, chooses unique ideas, and chooses creative ideas from a library of pre-generated ideas. The creative robot also engages in non-verbal interactions such as curiosity for new ideas and joy when it generates an idea. The other group interacts with the robot that exhibits noncreative behaviors, that is, chooses less number of ideas, chooses less unique ideas, and chooses less creative ideas.

We observed that participants who interacted with the creative robot exhibited more creative behaviors than the participants who interacted with the non-creative robot. Three measures of creativity were recorded, (1) the number of ideas that participants generated per droodle, (2) the number of unique themes they explored in their ideas, and (3) the creativity score of each idea. Creativity scores were ranked by blind coders following the Droodle Creativity Task coding system [23]. We observed that participants interacting with the creative robot scored significantly higher in creativity scores in all three measures as compared to participants who interacted with the non-creative robot. We conclude that children can model creativity from a social robot and inform Child-Robot Interaction Patterns that help foster creativity in children.

\section{BACKGROUND}

\section{Creativity}

There are several definitions of creativity, and what qualifies as creative behavior. From his work on the Structure of Intellect Model (SOI), Guilford suggested three basic components as factors of creativity: fluency, flexibility, and originality [19]. These three factors are still commonly acknowledged as basic components of divergent thinking. This work was influential in the development of the different definitions of creativity $[11,29,36,42]$, as well as development of standardized tests of creativity and divergent thinking $[8,10,29,32,1]$. Modern researchers describe creativity as the process of fluently generating artifacts or ideas, that are novel to the person, to the person's environment or to the world, that generate surprise, and that add value to the system [4]. For the purpose of this work, we define creativity as the process of generating ideas with fluency, that have novelty, and are valuable. Fluency refers to generating lots of different ideas to address the same problem. Novelty refers to generating ideas that are different from ideas of their peers, and as compared to their own previous ideas. This involves mapping all ideas presented by the individual over time, and ideas presented by all individuals. Value refers to generating ideas that add value to the problem being solved. This includes solving problems in the most optimized way, or using least time and resources to solve a problem.

Even though creativity remains challenging to measure empirically, researchers have developed creativity assessment tasks with coding systems that conceptualize and measure creativity empirically, such as, the Droodle Creativity Task [23] which ranks creativity based on participants' ability to generate surprising and witty titles for abstract images, the Unusual Uses Task [23] which ranks creativity based on participants' ability to think of alternate uses of an object, and the Candle Problem [13]. There have also been some attempts to measure creativity, typically in the form of standardized assessment tests [8] like the Torrance Test of Creativity Thinking [43] which is the most widely used test of creativity [12] and is the most referenced of all creativity tests [28]. Using measures of divergent thinking in figural and verbal expression, the TTCT [43] was one of the first to show that students' creativity began to decline around age 6 , slumped further in the fourth grade, but later showed a subsequent increase. This phenomenon became known as the fourth-grade slump. This decline in creativity has been found to be evident in as many as seven countries worldwide [45]. Smith and Carlsson [39, 40] found that after entering school, a slump in creativity occurred at ages 7 to 8 . One suggested reason for this slump is structured school curriculum, and lack of play based learning activities, such as that of kindergarten $[35,1]$. This drop in creativity, often referred to as the fourth grade slump, may reflect the pressure to conform that categorize educational settings [37]. Guilford [19] first noted how educational practices did not correlate well to children's creative thinking capacities.

External factors, such as children's social interactions with mentors and peers, can contribute to fostering creativity in children [25]. Amabile \& Gryskiewicz [2] and later Witt \& Beorkrem [48] identified the following "situational influences on creativity": freedom, autonomy, good role models and resources (including time), encouragement specifically for originality, freedom from criticism, and "norms in which innovation is prized and failure not fatal". Children's motivation and personality interact with the environment and components of the creative process to enhance the development of creativity in a child [11]. Creativity is influenced by immediate classroom environment, and fostered when activities are presented in 
permissive and game-like fashion [46]. Other creators in children's environment including teachers and peers also act as potential models for creativity [37].

Artificial embodied agents such as social robots have been studied to be effective pedagogical tools for young children leading to both cognitive and affective gains [3]. Previous studies have also demonstrated how children model a robotic peer's learning behaviors such as curiosity, perseverance and growth mindset [31]. In this work, we explore if interacting with social robots that exhibit creative behaviors in a play based environment can help children think more creatively.

\section{Robots and Creativity}

The use of robots in the classroom to foster creativity in children is not new. Lego Mindstorms, Bots Alive, Cozmo, Pop bots [5, 9, 26, 47] are examples of robot construction kits aimed to teach children about robotics and allow for creative expression. Alves-Oliveria et al. [1] built Yolo, a robot to be used by children as a tool to boost new ideas and stimulate their creativity. These works, however, uses the robot as a toolkit that children use to create, rather than as an agent that children interact with. Fischer and colleagues [14] embedded a software agent into an architectural design tool. The agent offered critiquing statements to promote a reflective design practice. Jung et al. [21] demonstrated how having a reflective conversation with an embodied artifact in the making positively influenced the learning process. Kahn et al. [24] explored if a social robot helps engage adults in creative tasks. They categorized the robot's interactions with people into several interaction patterns - Introduction, defining the creative space, evaluating similar experiences, forming an inventory of ideas, reflecting on intuition, pushing the limits, consider alternatives, building on foundational work, validating decision, engaging in creative conflict, and breaking loose. They found that participants engaged in creativity tasks longer and provided almost twice the number of creative expressions in the presence of the robot as compared to a PowerPoint presentation. These results help us understand that a robot's social interaction has the potential to motivate adults to come up with creative expressions. However, no such work has been conducted for analyzing the effect of robots' social interactions on children's creativity.

Social robots have previously been used as learning tools to foster positive learning behaviors, such as curiosity [18, 31], growth mindset [31], grit, persistence, and attentiveness [3]. Several studies have looked at how children model a social robot's learning behavior, such as curiosity or growth mindset. The physical presence of a robot tutor increases cognitive learning gains [27]. Gordon et al. demonstrated how children catch curiosity from a curious robot, and exhibit greater curiosity related behaviors such as question asking while interacting with a curious robot as compared to a non-curious robot [18]. Park et al. demonstrated how a robot exhibiting growth mindset can help foster a growth mindset in young children [31]. In a similar theme, in this work, we aim to explore if a robot exhibiting creative thinking can help foster creativity in young children. We make use of a game based interaction since play fosters children's creativity [30]. We suggest a set of novel verbal and non-verbal interactions as well as game action selection strategies of social robots that exhibit its creative behavior.

\section{EXPERIMENTAL SETUP}

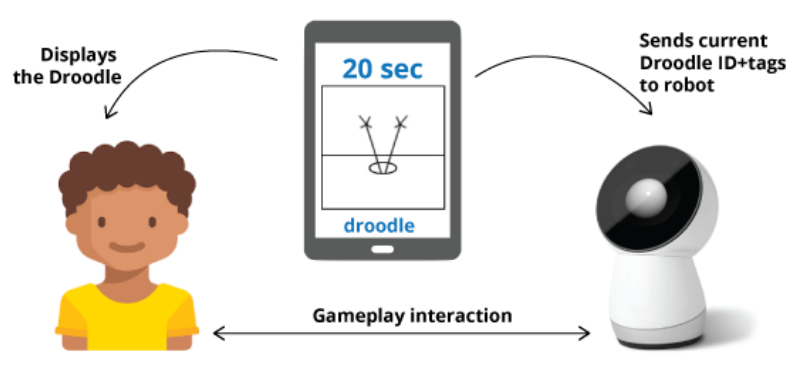

Figure 1. System Components. The child and the robot play the Droodle game collaboratively. The Droodles are displayed on the Android tablet. The tablet communicates the current Droodle ID and titles to the robot.

\section{Robotic Platform}

The social robot platform used for this study was the Jibo SDK [20]. Jibo is a socially expressive companion robot that has a three-axis body and a screen based face. Jibo uses a text-to-speech service for speech generation, and a speaker for sound output. Jibo also has a microphone to identify speech, cameras to detect images, and tactile sensors to detect touch. For the purpose of this study, we only make use of the microphone and the speaker. We make use of Jibo developer tool-kit's Android module for setting up communication between an Android tablet and the robot while they are on the same network. The game's logic lives on the Android application, which communicates with the Jibo platform (Fig 1).

\section{Droodle Creativity Game}

In order to understand how interacting with a robot influences children's creativity, we designed the Droodle Creativity Game that affords creativity as a gameplay behavior. The Droodle Creativity Game is inspired from the Droodle Creativity Task developed by Kahn et al. [23], to measure creativity. Participants are presented with a Droodle, a simple abstract drawing (Fig 2.b) that "comes into focus" (in a surprising way) with the addition of a clever title, and they are tasked with generating Droodle titles. The Droodle Task coding system provides a comprehensive guide to rank the titles provided by participants as 'nondroodle', 'low-', 'medium-', or 'high-droodle' by the coder based on their initial reaction, pattern matching, and categories and rationale. For instance, in (Fig 2.b) an example low-Droodle phrase would be '2 lines and 4 
circles', and an example high-Droodle phrase would be, ' $A$ bear climbing a tree'. In addition to these verbal interactions, the robot also showed multiple expressions of thought and curiosity, and expressed surprise and excitement upon coming up with creative titles. As a child friendly, game-like, fun activity, the Droodle Creativity Task is well situated to measure creativity in young children.

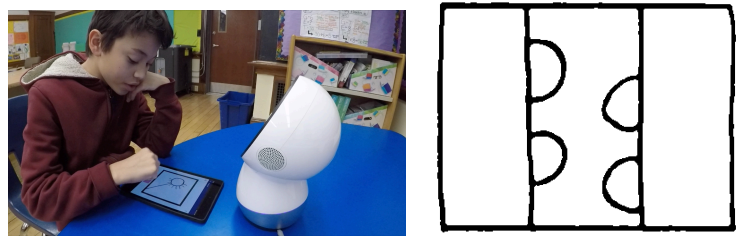

Figure 2. (a) Interaction Scene. A child is playing the Droodle Creativity Game on an Android Tablet with the social robot Jibo. (b) Example of a Droodle Image. 10 Droodles were used in the Droodle Creativity Game (5 per player).

For this study, we developed an interactive game inspired from the Droodle Creativity Task. The child and the robot take turns to play the game (Fig 2.a). The active player is presented with Droodles on a tablet screen and they come up with droodle title(s) in 30 seconds. Then the turn shifts to the other player until each player has played five turns each. Droodles used in the task were taken from Droodles: The Classic Collection [33], which also includes a library of droodle titles.

\section{Subjects}

51 participants in the 6-10-year-old age group were recruited for the study ( 24 female, 27 male). All 51 students completed the Torrance Test of Creative Thinking (TTCT) as a part of the pre-test activity. 2 participants were excluded from the analysis because of unclear audio transcription or incomplete audio files. 1 participant was excluded from the analysis because they were a non-native English speaker and participated in Spanish. Hence, we conducted the analysis on 48 participants' data (22 female, 26 male). The average age of the participants was 8.06 (S.D. $=1.78)$.

The subjects were recruited as a part of the [Anonymized] after-school activities program at the public schools in [Anonymized city]. 1 of the subjects had previously interacted with the robot used in the experiment. All students had basic knowledge of robotics and Artificial Intelligence taught to them as a part of another module of the after-school program. All participants and their guardians signed a consent to participate and for audio and video data collection.

\section{Study Protocol}

\section{Pre-test}

Participants were administered the first part of the verbal and figural module of the Torrance Test of Creative Thinking. The purpose of conducting the TTCT was to divide the participants in 2 balanced groups such that the mean and standard deviation of the two groups' creativity scores are similar (Table 1). The two groups were also balanced in terms of the participants' age and gender. One group interacted with the creative robot $(\mathrm{C}+$ condition $)$ and the other group interacted with the non-creative robot (C-robot).

$\begin{array}{lllll}\begin{array}{l}\text { Study } \\ \text { groups }\end{array} & \text { n } & \begin{array}{l}\text { TTCT } \\ \text { scores }\end{array} & \text { Gender } & \text { Age } \\ \begin{array}{l}\text { Creative } \\ \text { robot }(\mathrm{C}+)\end{array} & 24 & 42.16 \pm 7.17 & \begin{array}{l}\mathrm{F}=9, \\ \mathrm{M}=15\end{array} & 7.78 \pm 1.92 \\ \begin{array}{l}\text { Non- } \\ \text { creative }\end{array} & 24 & 40.66 \pm 6.01 & \begin{array}{l}\mathrm{F}=13, \\ \mathrm{M}=11\end{array} & 8.38 \pm 1.85 \\ \text { robot }(\mathrm{C}-) & & & & \end{array}$

Table 1. 48 participants' gameplay data was analyzed. Participants were divided in balanced groups based on TTCT scores, gender and age.

\section{Robot Introduction}

Children are first introduced to the robot by the experimenter as a peer that they would be playing the game with. The robot then engages in a short self-introduction and asks participants to introduce themselves, "Hi, my name is Jibo. I am a social robot. What is your name?"

\section{Task Introduction}

The robot explains the gameplay to the participants: 'I have a fun game for you today. Do you want to play with me? Do you like telling stories? We will look at some pictures, and try to think of funny and creative ideas about what they are. Let your imagination run wild. Come up with as many ideas as you can in 30 seconds. Let me show you an example.' The tablet app displays an example droodle selected from our library of Droodles, and the robot responds with a Droodle title. After the example Droodle, the robot asks, 'Are you ready to start the game?' The participants can respond with a 'Yes' or a 'No' both through speech and through buttons on the robot's screen. If the participants respond with a 'Yes', then the robot begins the game. If the participants respond with a 'No', then the robot proceeds to provide a second example Droodle. If the participants respond with 'No' more than once, experimenters intervene to help the participants understand the game until they are ready to play.

\section{Child-robot Co-play}

The robot and the participants take turns to play their rounds. In each round, the player is given a Droodle image on the tablet screen, and they have 30 seconds to say phrases describing the image. Participants can come up with as many phrases as they can. For every idea, the participants are required to press the idea button on the tablet screen, and records their idea. The tablet screen displays a timer and a counter for the number of phrases each player came up with. There is a total of 5 rounds. The rounds start with the robot's turn and the robot transfers the turns by using verbal phrases, 'Now it's your turn. Come up with as many ideas as you can.' and 'Now, it's my turn.'

\section{Robot Interactions}

The robot acts as a collaborative peer that participants play the Droodle game with. The robot takes the role of explaining the gameplay to the participants, and providing an example. 
During the robot's turn, the robot picks Droodles from the Droodle library, which contains a list of strings, and each string has been coded with (1) the number of themes explored, and (2) a creativity score (non-, low-, medium-, or high-droodle). The number of themes explore were calculated using Rake NLTK, a Natural Language Processing Library [34]. The creativity scores are calculated using the Droodle Task Coding system [23]. The robot also engages in verbal and non-verbal interactions expressing wonder, curiosity, excitement and surprise. For instance, when the robot is attempting to think of a droodle idea, it says, 'I wonder what else it can be', and has a curious expression in body posture and eye expression. During the child's turn, the robot engages in verbal and non-verbal expressions of pride, joy, and surprise. For instance, when the child generates droodle ideas, the robot praises the child by using phrases such as, 'Great job', 'I would not have thought of that.', 'You are doing great' and expresses pride and joy. While the verbal and non-verbal interactions of the robot are consistent across the study conditions, the responses that the robot generates in its turn differ based on the study condition $(\mathrm{C}+$ or $\mathrm{C}-)$.

\section{Data Collection}

Children's speech and video data was recorded. We made use of Google Cloud's Speech API [17], as well as manual transcribing by three researchers blind to the study to transcribe children's phrases.

\section{Study Conditions}

All participants were divided in two study condition groups, one that interacted with the creative robot and one that interacted with the non-creative robot. The groups were divided such that the participants in the two groups were balanced in terms of their mean and standard deviations of TTCT scores, age and gender (Table 1). The robot exhibits high or low creativity through gameplay and through verbal and nonverbal behaviors. We use Boden's [4] framework of creativity to design creative behaviors in gameplay, and Kahn et al.'s Droodle Task Coding system [23] to determine the creativity of Droodle titles.

\section{Creative Robot}

Gameplay:

- Fluency. The robot generated 4 - 5 ideas per Droodle.

- Novelty. The robot explores 3 or more different themes in the ideas generated.

- Value. The robot picks ideas from the library of titles that are tagged medium or high in droodle creativity.

\section{Non-Creative Robot}

Gameplay:

- The robot generated 1-2 ideas per Droodle.

- The robot explores 1-3 different themes in the ideas generated.
- The robot picks ideas from the library of titles that are tagged low or medium in droodle creativity. Low/medium-Droodle title phrases often include very literal descriptions of a picture.

\section{Hypotheses}

We hypothesize that the social robot's gameplay behavior can be modelled by children, and that children can learn creative thinking from a social robot. We form our hypothesis in these parts:

- H1: Participants interacting with the creative robot $(\mathrm{C}+)$ generate more number of ideas that participants interacting with the non-creative robot $(\mathrm{C}-)$

- H2: Participants interacting with the creative robot $(\mathrm{C}+)$ explore more themes of ideas than participants interacting with the non-creative robot $(\mathrm{C}-)$

- H3: Participants interacting with the creative robot $(\mathrm{C}+)$ generate more creative ideas than participants interacting with the non-creative condition (C-).

\section{Data Analysis}

\section{Creativity Measures}

Participants' creativity was measured using the Droodle Creativity Game in three parts:

- Fluency. The number of ideas that the participants generated.

- Novelty. The number of unique themes explored through the ideas. Each idea is associated with theme tags, which include all concepts and keywords included in the idea.

- Value. The droodle creativity scores of the ideas generated. The standard metric for analyzing creativity of Droodle titles as suggested by Kahn et al [24] is used. Droodles are graded on the scale of $0,1,2$, or 3, mapping to non-droodle, low-droodle, medium-droodle, and high-droodle respectively.

For instance, one participant came up with the following ideas for the droodle image in round 1 (Fig 1): 'It's peppa pig'; 'It's peppa pig's hands'; and 'It's frog hands.' This can be analyzed as: Number of ideas $=3$; Unique themes = "peppa pig", "hands", "frogs"; Droodle scores: 2, 3, 2.

\section{Condition Analysis}

We calculated children's creativity measures (Fluency scores, Novelty scores, and Value of each Droodle title). We further calculated the mean and standard deviation of the Novelty and Value scores for every Droodle image for each participant. For instance, if for Droodle 1, the participant generated 3 ideas, we calculated the Novelty and Value as the mean score of the three individual Novelty and Value scores. We then conducted unpaired T-tests between the creative and non-creative study participants, in order to identify the differences between the two groups, for each of the three creativity scores. 


\section{RESULTS}

\section{H1: Number of Droodle Ideas}

In order to test hypothesis H1, we first analyzed the number of ideas generated by the participants in the two study conditions. We observed that participants who interacted with the robot expressing high levels of creativity $(\mathrm{C}+)$ generated significantly more overall ideas $\left(p<0.01^{* *}\right)$ as compared to the participants who interacted with the robot expressing low levels of creativity (C-) (Table 2, Figure 3). The overall scores were calculated as a mean of their scores across all the study conditions. While participants in the $\mathrm{C}+$ group generated a greater number of ideas for all 5 Droodles, the significance in this difference was maintained across 4 out of 5 Droodles (D1: $p<0.05^{*}$; D2: $p<0.01^{* *}$; D3: $p<$ $0.05^{*}$; D4: $p<0.01^{* *}$; D5: $p>0.05$ ) (Fig 4). Hence, we confirm hypothesis $\mathrm{H} 1$ that participants interacting with the creative robot $(\mathrm{C}+)$ generated more ideas that children interacting with the non-creative robot (C-).

$\begin{array}{lllllll}\text { SG } & \text { D1 } & \text { D2 } & \text { D3 } & \text { D4 } & \text { D5 } & \text { Overall } \\ \text { C+ } & 2.875 & 3.167 & 4.25 & 3.667 & 2.667 & 3.325 \\ & \pm 1.03 & \pm 1.52 & \pm 1.62 & \pm 1.78 & \pm 1.55 & \pm 1.16 \\ \text { C- } & 2.125 & 2.083 & 3.417 & 2.417 & 2.042 & 2.417 \\ & \pm 1.15 & \pm 1.1 & \pm 1.47 & \pm 1.13 & \pm 1.33 & \pm 0.96 \\ p & <0.05^{*} & <0.01 * * & <0.05^{*} & <0.01^{* *} & >0.05 & 0.01 \\ & & & & & & * *\end{array}$

Table 2. Average number of ideas expressed by the $\mathrm{C}+$ and $\mathrm{C}$ group for each droodle. Participants in the $\mathrm{C}+$ condition expressed significantly more ideas than participants in the $\mathrm{C}$ condition. Within each droodle, this difference was significant for Droodles D1, D2, D3, D4.

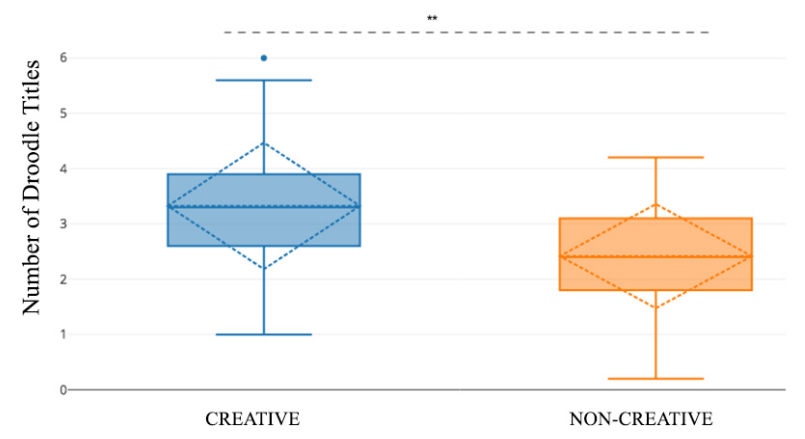

Figure 3. Participants in the $\mathbf{C}+$ condition generated significantly higher number of Droodle title ideas as compared to participants in the $\mathrm{C}$ - group $(\mathbf{p}<0.01 * *)$

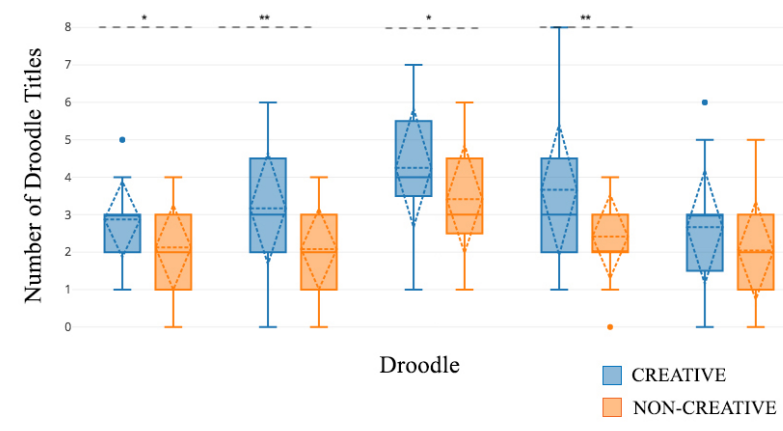

Figure 4. Participants in the $\mathbf{C}+$ group generated a greater number of title ideas per Droodle as compared to the $\mathbf{C}$ - group for each droodle. This difference was statistically significant for Droodles D1, D2, D3, D4.

\section{H2: Unique Themes Explored in Ideas}

In order to understand the novelty and range of Droodle title ideas that participants generated, we looked at the number of unique themes or concepts that constitute each idea. We used Rapid Automatic Keyword Extraction algorithm (Rake NLTK), a Natural Language Processing library to analyze the themes explored in each title [34]. RAKE is a domain independent keyword extraction algorithm which tries to determine key phrases in a body of text by analyzing the frequency of word appearance and its co-occurrence with other words in the text. We observed that participants who interacted with the robot expressing high levels of creativity explored significantly more overall unique themes $(p<$ $\left.0.01^{* *}\right)$ as compared to the participants who interacted with the robot expressing low levels of creativity (Table 3, Fig 5). Participants in the $\mathrm{C}+$ explored more themes in all five Droodle tasks, however this difference was significant for 3 out of 5 Droodles (D1: $p>0.05$; D2: $p<0.05^{*}$; D3: $p<$ $0.05^{*}$; D4: $p<0.01^{* *}$; D5: $p>0.05$ ) (Fig 6). Hence, we confirm hypothesis $\mathrm{H} 2$ that participants interacting with the creative robot $(\mathrm{C}+)$ explored more themes of ideas than children interacting with the non-creative robot (C-).

$\begin{array}{lllllll}\text { SG } & \text { D1 } & \text { D2 } & \text { D3 } & \text { D4 } & \text { D5 } & \begin{array}{l}\text { Over } \\ \text { all }\end{array} \\ \text { C+ } & 3.917 & 5.333 & 5.25 & 5.75 & 4.667 & 4.983 \\ & \pm 1.55 & \pm 2.66 & \pm 1.98 & \pm 1.7 & \pm 2.33 & \pm 1.25 \\ \text { C- } & 3.25 & 3.75 & 4.292 & 4.333 & 3.583 & 3.842 \\ & \pm 2.19 & \pm 2.32 & \pm 1.98 & \pm 2.12 & \pm 2.44 & \pm 1.66 \\ \boldsymbol{p} & >0.05 & <0.05 & <0.05 & <0.01 & >0.05 & <0.01 \\ & & * & * & * * & & * *\end{array}$

Table 3. Average number of unique themes explored by the $\mathrm{C}+$ and $\mathrm{C}$ - group for each droodle. Participants in the $\mathrm{C}+$ condition explored significantly more overall unique themes than participants in the $\mathrm{C}$ - condition. Within each droodle, this difference was significant for Droodles D2, D3, D4. 


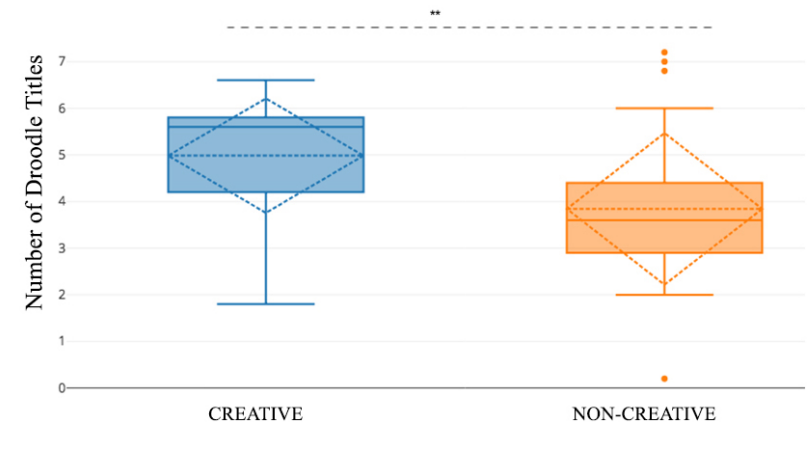

Figure 5. Participants in the $\mathbf{C}+$ condition explored significantly higher number of unique themes as compared to participants in the $\mathbf{C}$ - group $(\mathbf{p}<0.01 * *)$

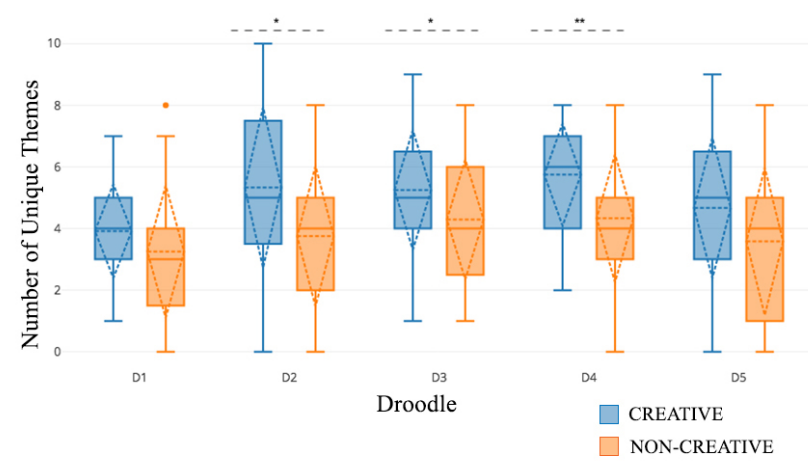

Figure 6. Participants in the $\mathrm{C}+$ condition explored more unique themes than participants in the $C$ - condition within each Droodle. The difference was significant for Droodles D2, D3, D4.

\section{H3: Creativity Scores}

All Droodle titles generated by the participants were coded as 'non-', 'low-', 'medium-' and 'high-droodle' by 3 blind coders. The coders were trained using the Droodle Creativity Task coding scheme. To determine inter-rater reliability between researchers, Cohen's kappa [7] was calculated using $67 \%$ of the coded transcripts coded independently by a team member after an initial coding by other two coders. Cohen's kappa was 0.82 which is within the range for substantial agreement considered acceptable for inter-rater reliability.

$\begin{array}{lllllll}\text { SG } & \text { D1 } & \text { D2 } & \text { D3 } & \text { D4 } & \text { D5 } & \begin{array}{l}\text { Over } \\ \text { all }\end{array} \\ \text { C+ } & 1.708 & 1.696 & 1.401 & 1.841 & 2.003 & 1.73 \pm \\ & \pm 0.41 & \pm 0.41 & \pm 0.41 & \pm 0.55 & \pm 0.39 & 0.21 \\ \text { C- } & 1.496 & 1.473 & 1.134 & 1.793 & 1.880 & 1.532 \\ & \pm 0.43 & \pm 0.46 & \pm 0.43 & \pm 0.59 & \pm 0.48 & \pm 0.25 \\ \boldsymbol{p} & <0.05^{*} & <0.05 & <0.05 & >0.05 & >0.05 & <0.01 \\ & & * & * & & & * *\end{array}$

Table 4. Average creativity scores per Droodle. Participants in the $\mathbf{C}+$ condition scored a significantly higher overall creativity score compared to the $C$ - condition. While participants in the $\mathrm{C}+$ condition scored higher than participants in the $\mathrm{C}$ - condition across all Droodles, the difference was significant for Droodles D1, D2, D3.

An analysis of overall creativity scores for every idea revealed that participants in the creative condition scored significantly higher in creativity score per title than participants in the non-creative condition $(p<0.01 * *)$ (Table 4, Fig 7). While the participants in the creative condition scored higher than participants in the non-creative condition for every droodle, the difference was statistically significant in 3 out of 5 Droodles (D1: $p<0.05^{*}$; D2: $p<$ 0.05*; D3: $p<0.05^{*}$; D4: $p>0.05$; D5: $p>0.05$ ) (Fig 8). Hence, we confirm H3 that participants interacting with the creative robot $(\mathrm{C}+)$ generated more creative ideas than children interacting with the non-creative condition $(\mathrm{C}-)$.

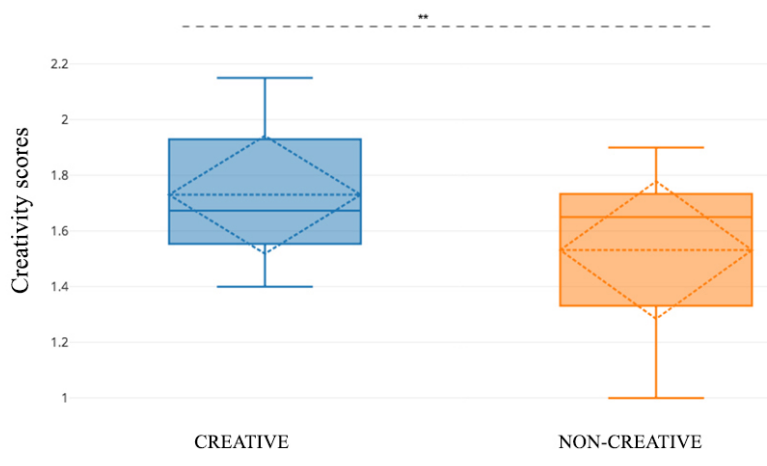

Figure 7. Participants in the $\mathrm{C}+$ condition scored a significantly higher overall creativity score as compared to participants in the $C$ - condition.

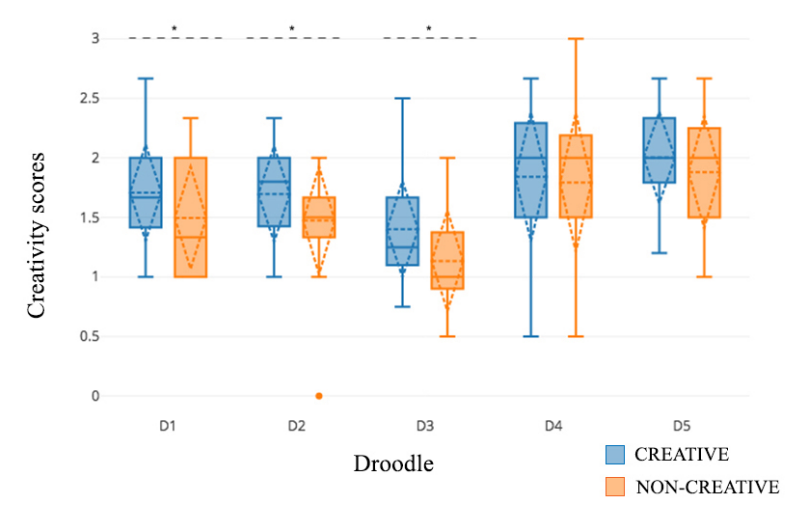

Figure 8. Participants in the $\mathrm{C}+$ condition scored higher creativity scores than participants in the $\mathrm{C}$ - condition within each Droodle. The difference was significant for Droodles D1, D2, D3.

\section{DISCUSSION}

In this work, we demonstrate how artificial creativity exhibited by a social robot during gameplay can be modelled by children, and in turn leads them into thinking more creatively in the context of the Droodle Creativity Game. This study involved participants in the 6-10-year-old age group playing the Droodle Creativity Game with a social robot expressing creative and non-creative behaviors. We observed that participants that interacted with the creative 
robot expressed more number of ideas, more diverse ideas, and highly creative ideas in the Droodle Creativity Game, as compared to participants that interacted with the noncreative robot. The positive effect of the robot's creative behavior on children's exhibited creativity in gameplay informs the design of pedagogical embodied tools to foster creativity. Previous work has discussed how advancements in Creativity Support Tools (CSTs) can help foster creativity in children [38]. Previous work has also discussed how adults interacting with a social robot that scaffolds for creativity enhance creative thinking [24]. This is the first work that demonstrates how autonomous social robots expressing creativity can be modelled by young children during play.

In classrooms, children not only learn cognitive skills from their teachers and peers, but also social-emotional skills such as curiosity, empathy, resilience, persistence and creativity. Social robots have already found a place in education as learning companions, and have shown to have learning benefits for young children due to their ability to facilitate personalized learning (a common struggle in large classroom sizes), and higher engagement (than non-embodied interfaces). Moreover, due to reduced hardware costs and easier availability, social robots have also entered domestic and classroom settings. While there is a huge body of existing research that focuses on using social robots to enhance children's cognitive learning benefits, there is very little research about how social robots can enhance children's creativity. It is imperative to think about designing these learning companion robots' social behaviors and understanding how children can model these behaviors. Previous studies have demonstrated how children mirror other robot behaviors such as curiosity and growth mindset. Hence, we ought to be mindful about designing technology to foster, and not hinder, positive learning behaviors. Our work demonstrates how a robot's verbal creativity skills are modelled by children in gameplay, and thus inform the behavioral design of social robots, such that they not only facilitate cognitive gains, but also focus on creative gains in children.

\section{CONCLUSION AND FUTURE WORK}

We have studied the effects of an autonomous social robot's verbal and nonverbal behavior on children's creativity as measured through the Droodle Creativity Game. We verified our hypotheses that children interacting with the highly creative robot generated more ideas, explored more themes of ideas, and generated more creative ideas as compared to children interacting with the non-creative robot. Social robots have known to be effective pedagogical agents that lead to both cognitive and affective gains in young children. Since robots are already being used in classroom settings as learning peers and personalized tutors, it is imperative to think about how these robots' behaviors can influence children's learning behaviors, such as creative thinking. We demonstrate how a robot's expressed creativity can be modelled by young children during gameplay. Embodied AI agents have the potential to use generative networks to express forms of creativity through generating media such as drawing, poetry, art styles, patterns, physical body movements, etc. This work opens up the opportunity to explore how these different forms of artificial creativity can be embedded in tools that children use, and help them be more creative.

It is important to note that while social robots are not the only way to provide this creativity support through behavioral modeling, they certainly are a compelling way. This technology is an affordable way to scale the support, and amplify and augment personalized social interaction.

One limitation of this work is that, even though the game is structured as a collaborative turn taking game interaction, one participant perceived it as a competitive game, and expressed a desire to 'beat' the robot. This competitive perception does not give the participant any incentive to generate more number of, or more creative ideas as compared to the robot. To address this concern, the games designed to afford creativity while interacting with a robot can be made even more collaborative, where the players' success is co-dependent, and they do not view the robot as a competitor. It is also important to note that while we found an overall significant difference in the creativity measures, the difference is not replicated in every droodle. Especially, in Droodle 5, none of the three effects are replicated. One reason for this effect could be gameplay lethargy, since we observed that participants often got tired till the $5^{\text {th }}$ round. However, in order to rule out the possibility of the specific Droodle image leading to this result, we must randomize the Droodle images and observe if the trend is similar. Lastly, this work is also limited to a narrow construct of creativity constrained to verbal responses that children generate in a game. Creativity encompasses a much wider array of behaviors that can be explored using other interactions such as figural tasks, or construction games. As a part of our future work, we aim to expand this study to other models of creativity and evaluate whether children model other forms of the social robot's creativity, such as figural creativity.

\section{ACKNOWLEDGMENTS}

We would like to thank all the participants who participated in the study, as well as their parents who consented to their participation. We also thank [ANONYMIZED] for their assistance with data collection. We would also like to extend our gratitude to the teachers of [ANONYMIZED] school for their cooperation and assistance.

\section{REFERENCES}

[1] Alves-Oliveira, P., Arriaga, P., Paiva, A., \& Hoffman, G. (2017, June). YOLO, a Robot for Creativity: A Co-Design Study with Children. In Proceedings of the 2017 Conference on Interaction Design and Children (pp. 423-429). ACM.

[2] Amabile, T. M., \& Gryskiewicz, N. D. (1989). The creative environment scales: Work environment 
inventory. Creativity research journal, 2(4), 231253.

[3] Belpaeme, T., Kennedy, J., Ramachandran, A., Scassellati, B., \& Tanaka, F. (2018). Social robots for education: A review. Science Robotics, 3(21), eaat5954.

[4] Boden, M. A. (2004). The creative mind: Myths and mechanisms. Routledge.

[5] Bots Alive. Dash Robotics. https://botsalive.com/

[6] Cognimates (2018). MIT Media Lanb. cognimates.me/

[7] Cohen, J. (1960). A coefficient of agreement for nominal scales. Educational and psychological measurement, 20(1), 37-46.

[8] Cooper, E. (1991). A critique of six measures for assessing creativity. The Journal of Creative Behavior, 25(3), 194-204.

[9] Cozmo (1999). Anki. https://www.anki.com/enus/cozmo

[10] Cramond, B. (2001). Interview with E. Paul Torrance on creativity in the last and next millennia. Journal of Secondary Gifted Education, 12, 116-121.

[11] Cropley, A. J. (1999). Creativity and cognition: Producing effective novelty. Roeper Review, 21, 253-260

[12] Davis, G. A. (1997). Identifying creative students and measuring creativity. In N. Colangelo \& G. A. Davis (Eds.), Handbook of gifted education (pp. 269-281). Needham Heights, MA: Viacom.

[13] Duncker, K. (1945). On problem solving. Psychological. lonographs. 58(5, Whole No. 270).

[14] Fischer, G., Nakakoji, K., Ostwald, J., Stahl, G., \& Sumner, T. (1993). Embedding computer-based critics in the contexts of design (pp. 157-164). Proceedings of CHI'93, New York, NY: ACM Press.

[15] Florida, R. (2004). The rise of the creative class and how it's transforming work, leisure, community and everyday life (Paperback Ed.).

[16] Gaut, B. (2010). The philosophy of creativity. Philosophy Compass, 5(12), 1034-1046.

[17] Google Cloud - Text to Speech. https:/cloud.google.com/speech-to-text/

[18] Gordon, G., Breazeal, C., \& Engel, S. (2015, March). Can children catch curiosity from a social robot?. In Proceedings of the Tenth Annual ACM/IEEE International Conference on HumanRobot Interaction (pp. 91-98). ACM.
[19] Guilford, J. P. (1950). Creativity. American Psychologist, 5, 444-445.

[20] Jibo SDK. https://www.jibo.com/

[21] Jung, M. F., Martelaro, N., Hoster, H., \& Nass, C. (2014, June). Participatory materials: having a reflective conversation with an artifact in the making. In Proceedings of the 2014 conference on Designing interactive systems (pp. 25-34). ACM.

[22] Kafai, Y. B. (2012). Minds in play: Computer game design as a context for children's learning. Routledge.

[23] Kahn Jr, P. H., Friedman, B., Severson, R. L., \& Feldman, E. N. (2005). CREATIVITY TASKS AND CODING SYSTEM-USED IN THE PLASMA DISPLAY WINDOW STUDY. University of Washington, Seattle, WA: The Information School, University of Washington.

[24] Kahn Jr, P. H., Kanda, T., Ishiguro, H., Gill, B. T., Shen, S., Ruckert, J. H., \& Gary, H. E. (2016, March). Human creativity can be facilitated through interacting with a social robot. In The Eleventh ACM/IEEE International Conference on Human Robot Interaction (pp. 173-180). IEEE Press.

[25] Kaufman, J. C., \& Sternberg, R. J. (Eds.). (2010). The Cambridge handbook of creativity. Cambridge University Press.

[26] Lego Mindstroms, The Lego Group. https:/www.lego.com/en-us/mindstorms

[27]Leyzberg, D., Spaulding, S., Toneva, M., \& Scassellati, B. (2012, January). The physical presence of a robot tutor increases cognitive learning gains. In Proceedings of the Annual Meeting of the Cognitive Science Society (Vol. 34, No. 34).

[28] Lissitz, R. W., \& Willhoft, J. L. (1985). A methodological study of the Torrance Tests of Creativity. Journal of Educational Measurement, $22,1-111$.

[29] Mumford, M. D. (2000). Something old, something new: Revisiting Guilford's conception of creative problem solving. Creativity Research Journal, 13, 267-276.

[30] Papert, S. (1980). Mindstorms: Children, computers, and powerful ideas. Basic Books, Inc.

[31] Park, H. W., Rosenberg-Kima, R., Rosenberg, M., Gordon, G., \& Breazeal, C. (2017, March). Growing growth mindset with a social robot peer. In Proceedings of the 2017 ACM/IEEE International Conference on Human-Robot Interaction (pp. 137-145). ACM. 
[32] Plucker, J. A., Beghetto, R. A., \& Dow, G. T. (2004). Why isn't creativity more important to educational psychologists? Potentials, pitfalls, and future directions in creativity research. Educational Psychologist, 39, 83-96.

[33] Price, R., \& Lovka, B. (2000). Droodles: The classic collection. Tallfellow Press.

[34] Rose, S., Engel, D., Cramer, N., \& Cowley, W. (2010). Automatic keyword extraction from individual documents. Text Mining: Applications and Theory, 1-20.

[35] Runco, M. A. (2000). Introduction to the special issue: Commemorating Guilford's 1950 presidential address. Creativity Research Journal, $13,245$.

[36] Runco, M. A. (2014). Creativity: Theories and themes: Research, development, and practice. Elsevier.

[37] Shaffer, D. W. (2006). How computer games help children learn. Macmillan.

[38] Shneiderman, B., Fischer, G., Czerwinski, M., Resnick, M., Myers, B., Candy, L., ... \& Jennings, P. (2006). Creativity support tools: Report from a US National Science Foundation sponsored workshop. International Journal of HumanComputer Interaction, 20(2), 61-77.

[39] Smith, G., \& Carlsson, I. (1985). Creativity in middle and late school years. International Journal of Behavioral Development, 8(3), 329-343.

[40] Smith, G.J.W. and Carlsson, L.M. (1990) The Creative Process: A Functional Model Based on Empirical Studies from Childhood to Middle Age. International Universities Press Inc., Madison.

[41] Sternberg, R. J., \& Grigorenko, E. L. (2000). Guilford's structure of intellect model and model of creativity: Contributions and limi- tations. Creativity Research Journal, 13, 309-316.
[42] Sung, I., \& Berland, M. (2017, June). Forest Friends Demo: A Game-Exhibit to Promote Computer Science Concepts in Informal Spaces. In Proceedings of the 2017 Conference on Interaction Design and Children (pp. 701-704). ACM.

[43] Torrance, E. P. (1966). The Torrance Tests of Creative Thinking-Norms-Technical Manual Research Edition-Verbal Tests, Forms A and BFigural Tests, Forms A and B. Princeton, NJ: Personnel Press

[44] "TORRANCE, E. (1967). UNDERSTANDING THE FOURTH GRADE SLUMP IN CREATIVE THINKING. FINAL REPORT.

[45] Torrance, E. P. (1968). A longitudinal examination of the fourth grade slump in creativity. Gifted Child Quarterly, 12(4), 195-199.

[46] Wallach, M. A., \& Kogan, N. (1965). Modes of thinking in young children: A study of the creativity-intelligence distinction.

[47] Williams, R. C. (2018). PopBots: Leveraging Social Robots to Aid Preschool Children's Artificial Intelligence Education.

[48] Witt, L. A., \& Beorkrem, M. N. (1989). Climate for creative productivity as a predictor of research usefulness and organizational effectiveness in an R\&D organization. Creativity Research Journal, 2(1-2), 30-40.

[49] W. A. Bainbridge, J. W. Hart, E. S. Kim, and B. Scassellati. The benefits of interactions with physically present robots over video-displayed agents. International Journal of Social Robotics, 3(1):41-52, 2011. 